\title{
Assessing the performance of Random Partitioning and K-Fold Cross Validation methods of evaluation of a Face Recognition System
}

\author{
Babatunde R. $\mathrm{S}^{1 *}$, Olabiyisi S. $\mathrm{O}^{2}$, Omidiora E. $\mathrm{O}^{2}$, Ganiyu R. $\mathrm{A}^{2}$, Isiaka R. $\mathrm{M}^{1}$ \\ ${ }^{1}$ Department of Computer Science \\ College of Information and Communication Technology \\ Kwara State University, Malete. Nigeria \\ ${ }^{2}$ Department of Computer Science and Engineering \\ Faculty of Engineering and Technology \\ Ladoke Akintola University of Technology, Ogbomoso. Nigeria \\ ronke.babatunde@kwasu.edu.ng; soolabiyisi@lautech.edu.ng; eoomidiora@lautech.edu.ng; \\ raganiyu@lautech.edu.ng; abdulrafiu.isiaka@kwasu.edu.ng
}

\begin{abstract}
Face recognition has been an active research area in the pattern recognition and computer vision domains due to its many potential applications in surveillance, credit cards, passport and security. However, the problem of correct method of partitioning the face data into train and test set has always been a challenge to the development of a robust face recognition system. The performance of the System was tested on locally acquired face database when the face database was randomly partitioned and when k-fold Cross Validation partition was used. The face database was captured under the condition of significant variations of rotation, illumination and facial expression. Quantitative evaluation experimental results showed that Random Sampling technique has a higher average recognition rate (96.7\%) than Cross Validation partition method (95.3\%). However, recognition time in Cross Validation is faster ( 0.36 secs) than that of Random Sampling (0.38 secs).
\end{abstract}

Keywords: Pattern Recognition, Cross Validation, k-fold, Random Sampling

\section{Background to the Study}

Face Recognition has being a broad area of research in the recent years. Its applications are continuously gaining demands due its requirements in person authentication, access control and surveillance systems amongst others (Thakur et.al, 2010). Human face cannot be directly used for building automated recognition due to high dimensionality of the face vectors and redundant information contained in the face vectors. The research in face recognition has recently focused on developing a face representation that is capable of capturing the relevant information in a manner which is invariant to facial expression and illumination. If features are inadequately represented, automated face recognition will not be effectively achieved. The classification and subsequent 
recognition time can be reduced by reducing dimension of the image data (Omidiora, 2006; Omidiora et.al, 2008). Effective dimensionality reduction encompasses feature extraction and feature selection.

Local Binary Pattern (LBP) feature extraction is a method proposed by (Ojala, et al, 2002). It has been used successfully in a number of applications. The standard way of using LBP-based feature extraction is to evenly distribute patches across an image, so that the whole image is covered. Each patch is of uniform size, and no patches overlap. LBP is then applied to each pixel of a patch resulting in a histogram representing the feature characteristics for that particular patch (Rose, Reena and Suruliandi, 2011). A feature vector is created by simply concatenating all of the histograms associated with each patch. These results in transformed features which are suitable for feature selection procedure to select optimal feature subsets (Babatunde et al, 2014). The primary purpose of feature selection is to choose a subset of available features, by eliminating features with little or no predictive information and also redundant features that are strongly correlated (Vieira et al,2010).

The ACO metaheuristic is characterized as being a distributed, stochastic search method based on the indirect communication of a colony of (artificial) ants, mediated by (artificial) pheromone trails. Ant Colony system involves simple agents (ants) that cooperate with one another to achieve an emergent, unified behaviour for the system as a whole, producing a robust system capable of finding high-quality solutions for problems with a large search space. The pheromone trails in ACO serve as distributed numerical information used by the ants to probabilistically construct solutions to the problem under consideration. The ants modify the pheromone trails during the algorithm's execution to reflect their search experience (Dorigo and Blum, 2005). The extraction and selection of the optimal features to represent a face image in a lower dimensional feature space to improve the performance of face recognition systems in terms of time and accuracy is significant.

In this paper, our main objective is to involve the use of the following parameters: training time, recognition time and recognition rate. The values of these parameters were compared on Random Sampling and k-fold Cross Validation. Statistical analysis of the two evaluation methods was carried out. LBP, ACO algorithms and Mahalanobis distance measure was employed for implementing a face recognition system. A locally acquired face database (LAFDAB) which contains photographs of 120 randomly selected individuals was captured with the aid of a 22x HD genx 300 Digital Camera.

\section{Methodology and Procedure of the Face Recognition System}

Typically, an RGB face image is normalized and preprocessed as shown in Figure 1a. The face of a subject is initially segmented into a number of uniform, evenly distributed regions that cover the entire image as shown in Figure 1c. LBP code of a pixel captures the structure of local brightness variations around it. The value is computed by sampling circularly around the selected pixel and setting 1-bits in the LBP value for each sample that is brighter than the center pixel using equation 1.

$$
\operatorname{LBP}\left(x_{c}, y_{c}-\sum_{n=0}^{7} s\left(i_{n}, i_{c}\right) 2^{n}\right)
$$

where $i_{c}$ corresponds to the grey value of the center pixel $\left(x_{c}, y_{c}\right)$, in to the grey values of the 8 surrounding pixels. The LBP patterns are obtained by circularly sampling around the center pixel. The effects of circular sampling are that each local neighbourhood is rotated into 
Babatunde R.S, Olabiyisi S.O, Omidiora E.O, Ganiyu R.A, Isiaka R.M; Assessing the performance of Random Partitioning and K-Fold Cross Validation methods of evaluation of a Face Recognition System. Advances in Image and Video Processing, Volume 3 No 6, December (2015); pp: 19-26

other pixel location and the sampling point on the circle surrounding the center point are rotated into a different orientation within each neighbourhood (Ahonen et. al, 2004).

The original RGB, preprocessed and segmented face images are shown in Figures $3 a, b$ and c. A feature vector describing the textural properties of a given area can be computed by calculating a histogram of the LBP code of each region located inside this area as shown in Figure $3 \mathrm{~d}$. The resultant texture feature is shown in Figure $3 \mathrm{e}$.

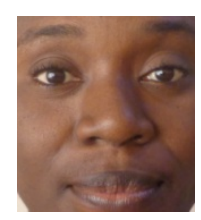

(a) Original image Orenting andin

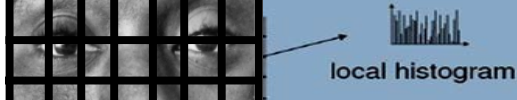

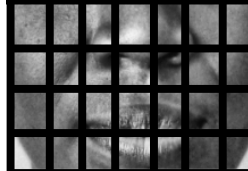

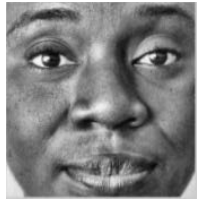

(b) preprocessed image

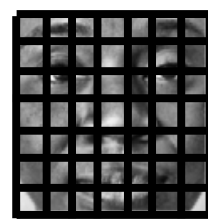

(c) Segmented face

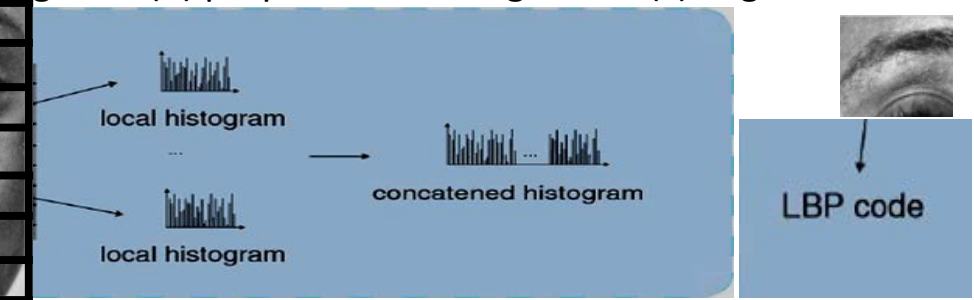

(d) LBP code of each region are the histograms

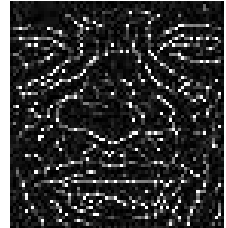

(e) Texture of face

Figure 1: sample face image and resultant LBP texture obtained

The matrix shown in Figure 2 a was obtained by thresholding the center pixel $c$, i.e. differences between $c$ and each of its neighbour pixels is calculated. Differences equal or greater than $c$ represents value 1 at the pixel position while difference less than $c$ represent 0 at pixel position as shown in the pattern matrix in Figure $2 b$.

\begin{tabular}{|l|l|l|}
\hline 88 & 93 & 104 \\
\hline 131 & 98 & 119 \\
\hline 96 & 89 & 110 \\
\hline
\end{tabular}

(a) Matrix of a region with pixel values

\begin{tabular}{|r|r|r|}
\hline 0 & 0 & 1 \\
\hline 1 & & 1 \\
\hline 0 & 0 & 1 \\
\hline
\end{tabular}

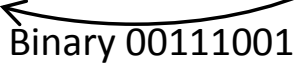

Decimal 57

(b)Pattern matrix obtained after thresholding the center pixel

Figure 2: The matrix of a sub-region and pattern matrix equivalent

After obtaining the pattern matrix the binary number associated with the pattern matrix is obtained in a clockwise manner as shown by the arrow. The decimal equivalent of the binary value is obtained using binomial weight and this gives the LBP of the region, represented by histogram. The output of LBP was converted to image data matrix to become input into ACO. 
The image data matrix will enable the feature selection task of ACO to be formulated by making the output of feature extraction ACO-suitable for selection of optimized feature subset. The image data matrix was created by converting the texture descriptors to double data format in order to set pixels (features) in a double array format so that the matrix of each image can be easily obtained and referenced (Babatunde et. al, 2015). The optimal feature subset is obtained using the probabilistic transition rule in equation 2

$$
P_{i, j}^{k}(t)=\frac{\left[\tau_{i, j}(t)\right]^{\alpha}\left[\eta_{i, j}\right]^{\beta}}{\sum_{j \in J^{k}}\left[\tau_{i, j}(t)\right]^{\alpha}\left[\eta_{i, j}\right]^{\beta}}, \text { ifi } \in J^{k}
$$

where $J^{k}$ is ant $\mathrm{k}^{\prime}$ s unvisited features, $\eta_{i}$ is the heuristic desirability of choosing feature $i, \tau_{i}$ is the pheromone value at feature $i, \alpha$ determine the importance of pheromone value $\beta$ determine the importance of heuristic information. $J_{i}^{k}$ is the neighborhood of ant $k$ when in node $i$. $\alpha$ determines the extent to which pheromone information is used as the ants build their solution. $B$ determines the extent to which heuristic information is used. The heuristic desirability for this experiment which is the measure of attractiveness of a feature (pixel) based on the local statistics of the image was obtained using the Pearson Product Moment Correlation. The heuristic desirability was obtained by computing the correlation between pairs of pixels. The ants move randomly over the face in a clique to construct a pheromone matrix. The size of the pheromone matrix for this experiment is the resolution of the cropped image (i.e. 70*70), which is arbitrarily chosen. The pheromone trail level and heuristic information are the two most important parameters which determine the success of solution construction in ACO. The process of construction of solution by the ants was carried out by adopting the probabilistic transition rule in equation (2). Once every pixel is visited, a subset of pixels is obtained which represents the optimal set of features (pixels) on the face image salient for face recognition. The resultant optimal feature selected by ACO is shown in Figure 3.

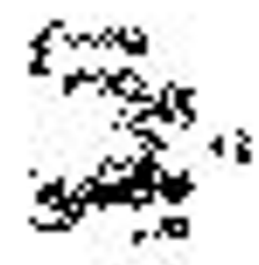

Figure 3: ACO subset image

The most discriminating features in a face pattern, selected by ACO from the face texture were encoded so that comparison between patterns can be made. The feature vectors corresponding to this subset of pixels were used for the recognition process. The Mahalanobis distance between these feature vectors and the test image vector was determined by comparing the covariance between the vectors of the test image and each of the trained images using equation 3.

$$
D_{i}^{\text {Mahalanobis }}=\sqrt{(x-\mu i)^{T} \sum_{i}^{-1}(x-\mu i)}
$$

where $\sum_{i}^{-1}$ represents the inverse of the covariance matrix of class I and $\mu i$ represents the mean of class I, $\mathrm{x}$ is the data point. The Flowchart of the dimensionality reduction process is shown in Figure 4 


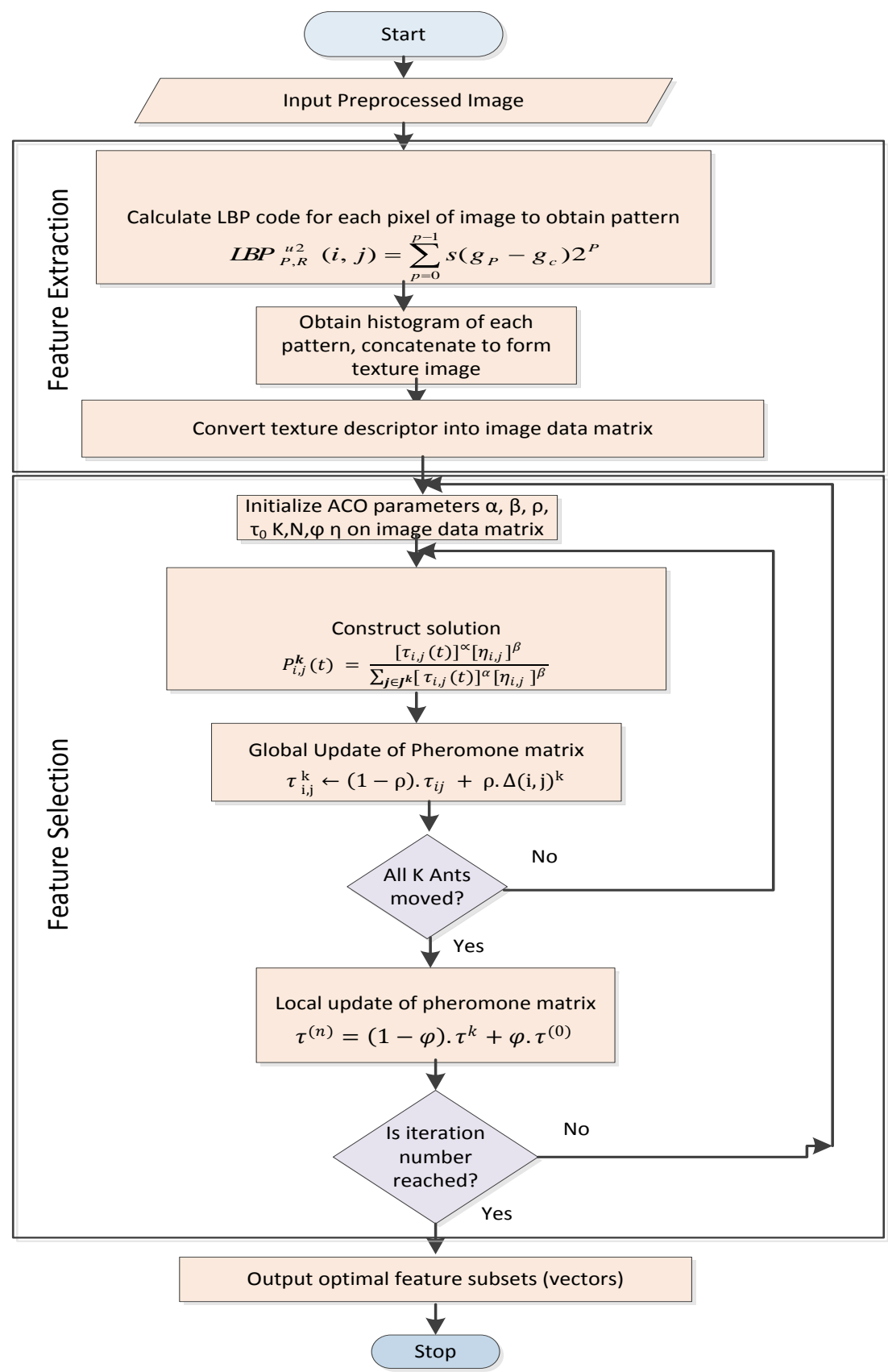

Figure 4: Flowchart of Feature Dimensionality Reduction for Face Recognition System

\section{Experimental Results and Discussion}

The dataset used in our experiment was composed of 720 coloured face images with 6 different images per subject for 120 individuals having a resolution of $1080 \times 1920$. The coloured faces were converted to gray scale images, cropped and resized to pixel resolution 70*70. These were carried out in MATLAB 2012R. The images were pre-processed to obtain uniform contrast using contrast limited adaptive histogram equalization technique. This was done because the images were captured locally under uncontrolled and various environmental conditions hence the need to stretch the contrast on the face images to obtain a uniform 
intensity of brightness. The configuration of the system used is Windows Professional Edition with a $2.4 \mathrm{GHz}$ Intel Core ' $\mathrm{i} 3$ processor, 64bit OS and $8 \mathrm{~GB}$ of RAM.

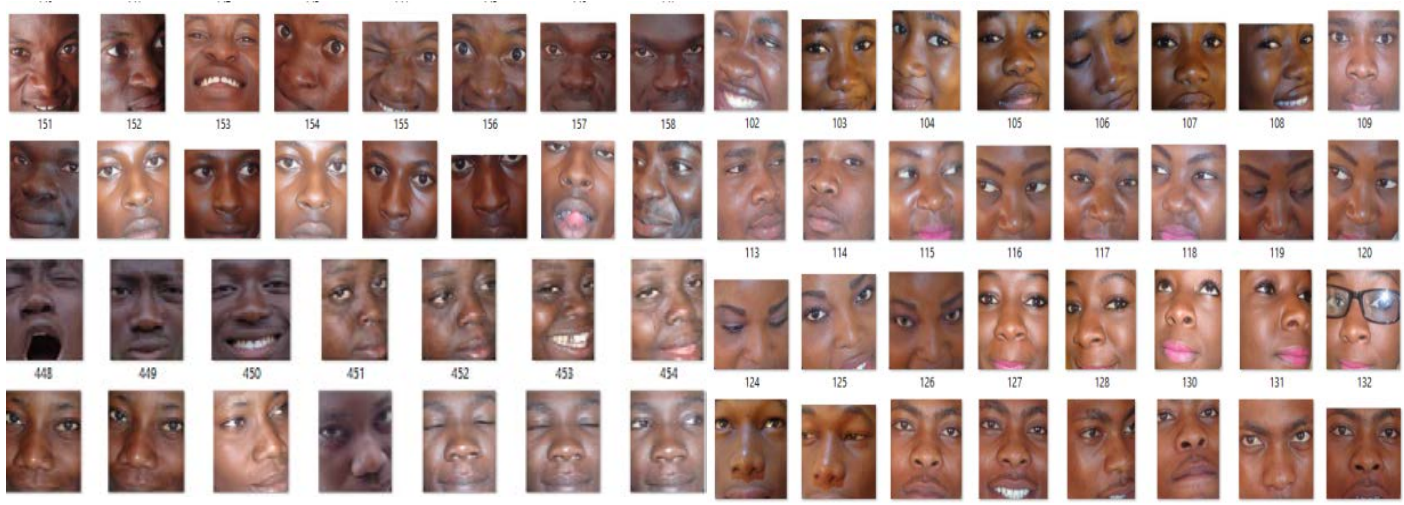

Figure 5: Sample faces from database

\subsection{Random partitioning of the database}

In this experimental strategy, the whole face images from LAFDAB (720) were randomly partitioned into training and testing sets. For each of the subjects, 4 images were randomly selected as training samples and the remaining 2 images as testing samples. A total of 480 of the 720 faces were used for training and the rest total of 240 facial images was used for testing so as to generate different training and testing sets. The training dataset after pre-processing was subjected to the dimensionality reduction technique to obtain the texture descriptor of the faces as well as optimized feature vectors. The optimal feature vectors were used for training. The result obtained is shown in Table 1

Table 1: Recognition Result of Random sampling of face data

\begin{tabular}{|l|c|}
\hline Parameter & Value \\
\hline Total Training Time (secs) & 417.16 secs \\
\hline Average Training Time (secs) & 0.87 secs \\
\hline Number of images in training set & 480 \\
\hline Total Recognition time (secs) & 90.06 secs \\
\hline Average Recognition time (secs) & 0.38 secs \\
\hline Number of images tested & 240 \\
\hline Number of images recognized & 232 \\
\hline Recognition rate & $96.7 \%$ \\
\hline
\end{tabular}

From the Table 1, the average training time obtained from the experiment was 0.87 secs, average recognition time was 0.38 secs and recognition rate was $96.7 \%$.

\subsection{Cross Validation Evaluation}

We also evaluated the performance of the face recognition system using Cross Validation method. In these experiments, the 720 images in the LAFDAB were divided into 6 folds due to the fact that there are 6 samples of each individual in the database. The images were first divided into 6 folds, with one image of a person in a fold. Hence, each fold consists 
Babatunde R.S, Olabiyisi S.O, Omidiora E.O, Ganiyu R.A, Isiaka R.M; Assessing the performance of Random Partitioning and K-Fold Cross Validation methods of evaluation of a Face Recognition System. Advances in Image and Video Processing, Volume 3 No 6, December (2015); pp: 19-26

of 120 images; each one image corresponds to a different person. At each experimental run, 5 folds were used to train and the remaining 1 fold was used for testing. Therefore, the training and testing sets consists of 600 and 120 images respectively in a particular experimental run. The recognition rates for all 6 runs were obtained. The results are shown in Table 2.

Table 2: Result of 6-fold Cross-Validation procedure

\begin{tabular}{|c|c|c|c|c|}
\hline Image fold & $\begin{array}{c}\text { Training } \\
\text { time(secs) }\end{array}$ & $\begin{array}{c}\text { Recognition } \\
\text { time(secs) }\end{array}$ & $\begin{array}{c}\text { Total Number of } \\
\text { images recognized }\end{array}$ & $\begin{array}{c}\text { Recognition } \\
\text { Rate\% }\end{array}$ \\
\hline Fold1 & 588.04 & 43.28 & 115 & 95.8 \\
\hline Fold2 & 578.92 & 43.01 & 117 & 97.5 \\
\hline Fold3 & 577.38 & 43.33 & 114 & 95.0 \\
\hline Fold4 & 581.16 & 42.89 & 116 & 96.7 \\
\hline Fold5 & 583.87 & 42.91 & 111 & 92.5 \\
\hline Fold6 & 582.41 & 43.15 & 113 & 94.1 \\
\hline $\begin{array}{c}\text { Average for 6 } \\
\text { folds }\end{array}$ & 589.5 & 43.09 & & 95.3 \\
\hline & $0.98 s e c s /$ image & $0.36 \mathrm{sec} /$ image & & \\
\hline
\end{tabular}

From Table 2, the average training time for the 6 folds was 589.50secs. Therefore, the average training time for the 600 face images in each training set is 0.98 secs per image. Similarly, the average recognition time obtained for the 6 folds was 43.09 secs; hence average recognition time per face image could be taken to be 0.36 secs. The average recognition rate obtained using 6 -fold cross validation method is $95.3 \%$.

\subsection{Evaluation of the Results of the two Methods}

Inferential Statistical analysis using Paired Sampled t-test was used to analyze the results obtained for Training Time, Recognition Time and Recognition Rate respectively for the two evaluation methods. The Paired Sampled t-test was performed on the null hypothesis $\left(\mathrm{H}_{0}\right)$ that there is significant difference between Random Sampling (RS) method and Cross Validation (CV) partition method against the alternative that there is no significant difference $\left(H_{1}\right)$, at $5 \%$ level of significance. The hypothesis is defined below;

$\mathrm{H}_{0}$ : There is significant difference between RS and CV method

$\mathrm{H}_{1}$ : There is no significant difference between RS and CV method

$x=[0.87,0.38,96.7]$ and $y=[0.98,0.36,95.3]$

$[h, p, c]=\operatorname{ttest}(x, y)$. The $p$-value obtained by performing the test was 0.4615 . Since $p$-value is greater than 0.05 , we therefore reject the null hypothesis, hence there is no significant difference between the two methods. This signifies that the choice of any of the two methods considered depends on the preference of the researcher involved. However, from the result obtained in the two experiments quantitatively, Random Sampling technique has a higher average recognition rate (96.7\%) than Cross Validation method. Additionally, the recognition time obtained using CV partition is smaller than RS by 0.02 , indicating a faster recognition time than RS, while the training time in CV is higher than RS.

\section{Conclusion}

In this research, the performance of Random Partitioning and k-fold Cross Validation methods of evaluation of a Face Recognition System was carried out. This was done to assess the effectiveness 
of employing any of the evaluation methods. The two methods performed well (and there was no significant difference in the performance of the two methods); hence the choice of any one depends on the preference of the researcher involved. Further research interest hopes to increase the number of folds in the Cross Validation, Kappa Statistics, as well as perform Leave-One-Out Cross Validation partitioning of data.

\section{REFERENCES}

[1]. Omidiora E. O,2006: A Prototype of Knowledge-Based System for Black Face Recognition using Principal Component Analysis and Fisher Discriminant Algorithms. Unpublished Ph. D Thesis, Department of Computer Science and Engineering, Ladoke Akintola University of Technology, Ogbomoso, Nigeria.

[2]. Dorigo, M. Stützle, T. (2002): The ant colony optimization metaheuristic: Algorithms applications and advances. In F. Glover and G. Kochenberger, editors, Handbook of Metaheuristics. International series in Operations Research and Management Science. Kluwer Academic Publishers. Vol. 57. pp. 251-285.

[3]. Dorigo, Marco., Blum, Christian. (2005): Ant colony optimization theory. A survey. Theoretical Computer Science Vol. 344 pp. 243 - 278. www.elsevier.com/locate/tcs

[4]. Babatunde R. S, Olabiyisi S.O, Omidiora E.O, Ganiyu R. A. (2014): Feature Dimensionality Reduction using a Dual Level Metaheuristic Algorithm International Journal of Applied Information Systems. Vol. 7(1). pp. 49-52.

[5]. Ojala T., Pietikäinen M., and Mäenpää. T. Multiresolution gray-scale and rotation invariant texture classification with local binary patterns. (2002): IEEE Transactions on Pattern Analysis and Machine intelligence, Vol 24. pp. 971-987

[6]. Thakur, S. Sing J. K., Basu D. K., Nasipuri, M., Kundu M. (2010): Face Recognition using Principal Component Analysis and RBF Neural Networks. IJSSST, Vol. 10(5).

[7]. Omidiora E.O., Fakolujo O.A., Ayeni R.O., Olabiyisi S.O., and Arulogun O.T. (2008): Quantitative Evaluation of Principal Component Analysis and Fisher Discriminant Analysis Techniques in Face images. Journal of Computer and its Applications. Vol.15 (1). pp. 22-37.

[8]. Rose R. Reena and Suruliandi A. (2011): Improving Performance of Texture Based Face Recognition Systems by Segmenting Face Region. International Journal of Network Security, Vol. 02, No. 03.Pp 23-27.

[9]. Babatunde R. S, Olabiyisi S. O, Omidiora E. O, Ganiyu R. A. (2015): Local Binary Pattern And Ant Colony Optimization Based Feature Dimensionality Reduction Technique For Face Recognition System. British Journal of Computer Science and Mathematics(Article in Press)

[10]. Kashef S., Nezamabadi-pour H. (2014). An advanced ACO algorithm for feature subset selection. Neurocomputing. pp. 1-9. http://dx.doi.org/10.1016/i.neucom.2014.06.067i 
Babatunde R.S, Olabiyisi S.O, Omidiora E.O, Ganiyu R.A, Isiaka R.M; Assessing the performance of Random Partitioning and K-Fold Cross Validation methods of evaluation of a Face Recognition System. Advances in Image and Video Processing, Volume 3 No 6, December (2015); pp: 19-26

[11]. Kavita, Chawla H.S. and Saini J.S. (2011): Parametric comparison of Ant colony optimization for edge detection problem. International Journal of Computational Engineering \& Management, 13:54-58

[12]. SodhiKuldeep Singh and Lal Madan. (2013): Comparative Analysis of PCA-based Face Recognition System using different Distance Classifiers. International Journal of Application on Innovation in Engineering and Management. Vol 2(7):341-348. 